\title{
PERMUTATION GROUPS AND PERIODICITY OF SYSTEMS OF DIFFERENCE EQUATIONS
}

\author{
Alaa E. Hamza ${ }^{\dagger, 1}$, Nasr Zeyada ${ }^{\dagger, 2}$ and A. M. Ahmed ${ }^{\dagger \dagger, 3}$ \\ ${ }^{\dagger}$ Department of Mathematics, Faculty of Science, University of Jeddah, Jeddah, 21589, Saudi Arabia. \\ ${ }^{\dagger}$ Department of Mathematics, Faculty of Science, Cairo University, Giza, Egypt. \\ ${ }^{1}$ E-mail: hamzaaeg2003@yahoo.com \\ ${ }^{2}$ E-mail: nzeyada@gmail.com \\ ${ }^{\dagger \dagger}$ Department of Mathematics, Faculty of Science, Jouf University, Skaka 2014, Aljouf, Saudi Arabia. \\ ${ }^{3}$ E-mail: ahmedelkb@yahoo.com \& amaahmed@ju.edu.sa
}

Received 10/8/2017 Revised Accepted 23/12/2017

\section{Abstract}

Let $k \in \mathbb{N}, \mathbb{Z}_{k}=\{1,2, \ldots, k\}$ and $S_{k}$ be the group of all permutations on $\mathbb{Z}_{k}$. Let $\pi \in S_{k}$ be of order $l$ and $f_{i}$ be a function from a nonempty set $X$ into itself, $i=1, \ldots, k$. In this paper, we show that a sufficient condition for a system of difference equations

$$
x_{n+1}^{(1)}=f_{1}\left(x_{n-s}^{(\pi(1))}\right), \quad x_{n+1}^{(2)}=f_{2}\left(x_{n-s}^{(\pi(2))}\right), \ldots x_{n+1}^{(k)}=f_{k}\left(x_{n-s}^{(\pi(k))}\right), n \in \mathbb{Z}^{\geq 0},
$$

to be periodic with a period $d$ is that each difference equation

$$
y_{n+l(s+1)-s}=g_{i}\left(y_{n-s}\right), n \in \mathbb{Z}^{\geq 0},
$$

is periodic, $i=1, \ldots, k$, with a period $d_{i}$ that divides $d$. Here, $g_{i}$ is defined by

$$
g_{i}=f_{i} f_{\pi(i)} \ldots f_{\pi^{l-1}(i)}, i=1, \ldots, k .
$$

Finally, the periodicity of many systems of rational difference equations is established.

Keywords: Systems of difference equations, permutation groups, periodicity.

Mathematics Subject classification: 20B99,39A10,39A23.

${ }^{1}$ On leave from Department of Mathematics, Faculty of Science, Al-Azhar University , Nasr City (11884), Cairo, Egypt.

\section{Introduction and preliminaries}

The theory of permutation groups is important to diverse area of mathematics such as Galois theory, invariant theory, the representation theory of Lie groups, and combinatorics. See for instance [1]. In general, the theory of abstract groups plays an important part in present day mathematics and science. Groups arise in a bewildering number of apparently unconnected subjects. Thus they appear in algebra and analysis, in geometry and topology, in crystallography and quantum mechanics, in physics and chemistry, and even in biology. See [2] , [3]. In this paper, as another application of the theory of permutation groups, we investigate the periodicity of systems of difference equations. Recently, there has been a great interest in difference equations, because they describe naturally many real-life problems in biology, ecology, genetics, psychology, sociology, and so forth. The authors in [4] investigated the periodicity of the system

$$
x_{n+1}^{(1)}=\frac{x_{n}^{(2)}}{x_{n}^{(2)}-1}, \quad x_{n+1}^{(2)}=\frac{x_{n}^{(3)}}{x_{n}^{(3)}-1}, \ldots x_{n+1}^{(k)}=\frac{x_{n}^{(1)}}{x_{n}^{(1)}-1}, n \in \mathbb{Z}^{\geq 0},
$$


as well as the periodicity of the system

$$
x_{n+1}^{(1)}=\frac{x_{n}^{(k)}}{x_{n}^{(k)}-1}, \quad x_{n+1}^{(2)}=\frac{x_{n}^{(1)}}{x_{n}^{(1)}-1}, \ldots x_{n+1}^{(k)}=\frac{x_{n}^{(k-1)}}{x_{n}^{(k-1)}-1}, n \in \mathbb{Z}^{\geq 0} .
$$

For some related results about periodicity, we refer the reader to the interesting papers [5-9]. Motivated by systems (1.1) and (1.2), we investigate the periodic character of the general system

$$
x_{n+1}^{(1)}=f_{1}\left(x_{n-s}^{(\pi(1))}\right), \quad x_{n+1}^{(2)}=f_{2}\left(x_{n-s}^{(\pi(2))}\right), \ldots x_{n+1}^{(k)}=f_{k}\left(x_{n-s}^{(\pi(k))}\right) .
$$

Here, $\pi \in S_{k}$ and $f_{i}$ is a certain function from a non-empty set into itself, $i=1, \ldots, k$. At the end of this paper, the periodicity of many systems of rational difference equations is investigated. It is well-known that for any permutation $\pi \in S_{k}$, there is a natural number $l$ such that the property $\pi^{l}=I$ holds, where $I$ is the identity permutation and $\pi^{l}$ is the composition of $\pi$ with itself $l$-times. The smallest $l$ for which this property holds is called the order of $\pi$.

Definition 1.1. A system (1.3) is called periodic with period d if every solution $\left(x_{n}^{(1)}, x_{n}^{(2)}, \ldots, x_{n}^{(k)}\right)$ satisfies

$$
x_{n+d}^{(i)}=x_{n}^{(i)}, n \in \mathbb{Z}^{\geq 0}, i \in \mathbb{Z}_{k} .
$$

Definition 1.2. A function $f: X \rightarrow X$ is called involution (resp. idempotent) if $f^{2}=I\left(\right.$ resp. $\left.f^{2}=f\right)$. Here, $I$ is the identity function.

\section{Main results}

Let $\pi \in S_{k}$ be a permutation of order $l, X$ be any non-empty set and $f_{i}: X \rightarrow X, i=1, \ldots, k$. In this section we investigate the periodicity of the system

$$
x_{n+1}^{(1)}=f_{1}\left(x_{n-s}^{(\pi(1))}\right), \quad x_{n+1}^{(2)}=f_{2}\left(x_{n-s}^{(\pi(2))}\right), \ldots x_{n+1}^{(k)}=f_{k}\left(x_{n-s}^{(\pi(k))}\right)
$$

in terms of the periodicity of each of the difference equations

$$
y_{n+l(s+1)-s}=g_{i}\left(y_{n-s}\right), n \geq 0
$$

where $g_{i}$ is defined by

$$
g_{i}=f_{i} f_{\pi(i)} \ldots f_{\pi^{l-1}(i)}, i=1, \ldots, k .
$$

We need the following lemma to prove our main result.

Lemma 2.1. (i) If a $k$-tuple $\left(x_{n}^{(1)}, x_{n}^{(2)}, \ldots, x_{n}^{(k)}\right)$ is a solution of system (2.1), then it satisfies the following equations

$$
x_{n+l(s+1)-s}^{(i)}=f_{i} f_{\pi(i)} \ldots f_{\pi^{r}(i)}\left(x_{n+l(s+1)-s-(r+1)(s+1)}^{\left(\pi^{r+1}(i)\right)}\right), i \in \mathbb{Z}_{k},
$$

for every $r \in \mathbb{Z}^{\geq 0}$.

(ii) Assume that $l$ is even. If a $k$-tuple $\left(x_{n}^{(1)}, x_{n}^{(2)}, \ldots, x_{n}^{(k)}\right)$ is a solution of system (2.1), then it satisfies the following equations

$$
x_{n+\frac{l}{2}(s+1)-s}^{(i)}=f_{i} f_{\pi(i)} \ldots f_{\pi^{r}(i)}\left(x_{n+\frac{l}{2}(s+1)-s-(r+1)(s+1)}^{\left(\pi^{r+1}(i)\right)}\right), i \in \mathbb{Z}_{k},
$$

for every $r \in \mathbb{Z}^{\geq 0}$.

Proof. (i) Relation (2.3) is true at $r=0$. Assume that relation (2.3) is true for a fixed $r$. We have

$$
\begin{aligned}
x_{n+l(s+1)-s}^{(i)} & =f_{i} f_{\pi(i)} \ldots f_{\pi^{r}(i)}\left(x_{n+l(s+1)-s-(r+1)(s+1)}^{\left(\pi^{r+1}(i)\right)}\right) \\
& =f_{i} f_{\pi(i)} \ldots f_{\pi^{r}(i)} f_{\pi^{r+1}(i)}\left(x_{n+l\left(\pi^{r+1}\right)-s-(r+1)(s+1)-(s+1)}^{\left(\pi^{r+2}\right)}\right) \\
& =f_{i} f_{\pi(i)} \ldots f_{\pi^{r+1}(i)}\left(x_{n+l(s+1)-s-(r+2)(s+1)}^{\left(\pi^{r+2}(i)\right)}\right), n \geq 0 .
\end{aligned}
$$


(ii) can be shown similarly.

As a direct consequence, putting $r=l-1$, we get the following result

\section{Corollary 2.2.}

(i) If a $k$-tuple $\left(x_{n}^{(1)}, x_{n}^{(2)}, \ldots, x_{n}^{(k)}\right)$ is a solution of system (2.1), then $x_{n}^{i}$ satisfies the equation

$$
y_{n+l(s+1)-s}=g_{i}\left(y_{n-s}\right), n \geq 0,
$$

for each $i$.

(ii) Assume that $l$ is even. If a $k$-tuple $\left(x_{n}^{(1)}, x_{n}^{(2)}, \ldots, x_{n}^{(k)}\right)$ is a solution of system $(2.1)$, then $x_{n}^{i}$ satisfies the equation

$$
y_{n+\frac{l}{2}(s+1)-s}=g_{i}\left(y_{n-\frac{l}{2}(s+1)-s}\right), n \geq 0
$$

for each $i$.

To arrive at our main result, we need to check the following Lemma

Lemma 2.3. Let $g: X \rightarrow X$.

(i) A solution of the equation

$$
y_{n+l(s+1)-s}=g\left(y_{n-s}\right), n \in \mathbb{Z}^{\geq 0}
$$

is given by

$$
y_{(m+1)(s+1) l+r-s}=g^{m+1}\left(y_{r-s}\right), r=0, \ldots,(s+1) l, m \in \mathbb{Z}^{\geq 0} .
$$

(ii) Assume that $l$ is even. A solution of the equation

$$
y_{n+\frac{l}{2}(s+1)-s}=g\left(y_{n-\frac{l}{2}(s+1)-s}\right), n \in \mathbb{Z}^{\geq 0}
$$

is given by

$$
y_{m(s+1) \frac{l}{2}+r-s}=\left\{\begin{array}{l}
g^{\frac{m}{2}}\left(y_{r-s}\right), \quad \text { m upiseven }, \\
g^{\frac{m+1}{2}}\left(y_{r-s-(s+1) \frac{l}{2}}\right), \quad m \text { upisodd } .
\end{array}\right.
$$

Proof. (i) Relation (2.6) is true when $m=0$. Assume it is true for a fixed $m$. Simple calculations show that

$$
\begin{aligned}
y_{(m+2)(s+1) l+r-s} & =g\left(y_{(m+2)(s+1) l+r-s-(s+1) l}\right) \\
& =g\left(y_{(m+1)(s+1) l+r-s}\right) \\
& =g\left(g^{m+1}\left(y_{r-s}\right)\right) \\
& =g^{m+2}\left(y_{r-s}\right) .
\end{aligned}
$$

(ii) Relation (2.8) is true at $m=0$ and $m=1$. Let $m$ be a fixed natural number and the relation is true for every natural number less than $m$. For the case where $m$ is even, we have

$$
\begin{aligned}
y_{m(s+1) \frac{l}{2}+r-s} & =g\left(y_{m(s+1) \frac{l}{2}+r-s-(s+1) l}\right) \\
& =g\left(y_{(m-2)(s+1) \frac{l}{2}+r-s}\right) \\
& =g\left(g^{\frac{m-2}{2}}\left(y_{r-s}\right)\right) \\
& =g^{\frac{m}{2}}\left(y_{r-s}\right) .
\end{aligned}
$$

That is relation (2.8) is true for $m$, when $m$ is even. The case where $m$ is odd can be treated similarly. 
Now, we are ready to prove our main results.

Theorem 2.4. A sufficient condition for the system (2.1) to be periodic with a period $d$ is that each difference equation (2.5.i) ( resp. (2.6.i) when $l$ is even ) is periodic, with a period $d_{i}$ such that $d=l . c . m\left(d_{1}, \ldots, d_{k}\right)$, the least common multiple of $d_{1}, \ldots, d_{k}$.

Proof. Let $\left(x_{n}^{(1)}, x_{n}^{(2)}, \ldots, x_{n}^{(k)}\right)$ be a solution of system $(2.1)$. Then $x_{n}^{(i)}$ is a solution of $(2.5 . i)$ ( resp. (2.6.i) when $l$ is even ). The periodicity of $x_{n}^{(i)}$ with period $d_{i}, i=1, \ldots, k$ imply the periodicity of system $(2.1)$ with period $d=l . c . m\left(d_{1}, \ldots, d_{k}\right)$, the least common multiple of $d_{1}, \ldots, d_{k}$.

Theorem 2.5. If $f_{i}$ is an involution on $X$ for each $i$ such that $f_{i} f_{j}=f_{j} f_{i}, i, j \in \mathbb{Z}_{k}$, then system (2.1) is periodic with period $2 l(s+1)$.

Proof. Let $\left(x_{n}^{(1)}, x_{n}^{(2)}, \ldots, x_{n}^{(k)}\right)$ be a solution of system (2.1). Then $x_{n}^{i}$ is a solution of equation (2.5.i) for each $i$. By Lemma 2.3 , this solution is given by

$$
y_{(m+1)(s+1) l+r-s}=g_{i}^{m+1}\left(y_{r-s}\right), r=0, \ldots,(s+1) l, m \in \mathbb{Z}^{\geq 0} \text {. }
$$

The assumptions of the theorem imply that $g_{i}^{2}$ is the identity function for each $i$. We deduce that each equation (2.5. $i$ ) is periodic with period $2 l(s+1)$. Indeed, we conclude that

$$
\begin{aligned}
x_{(m+3)(s+1) l+r-s}^{(i)} & =g_{i}^{m+3}\left(x_{r-s}^{(i)}\right) \\
& =g_{i}^{m+1}\left(x_{r-s}^{(i)}\right) \\
& =x_{(m+1)(s+1) l+r-s}^{(i)}
\end{aligned}
$$

Theorem 2.6. If $f_{1}=\cdots=f_{k}=f$ is an involution on $X$ and $l$ is even, then system (2.1) is periodic with period $l(s+1)$.

Proof. The hypothesis implies that $g_{i}=f^{l}=I, i \in \mathbb{Z}_{k}$ and equation (2.5.i) is periodic with period $l(s+1)$ for each $i$.

Theorem 2.7. Assume that $f_{i}$ is an idempotent on $X$ for each $i$ such that $f_{i} f_{j}=f_{j} f_{i}, i, j \in \mathbb{Z}_{k}$. Then system $(2.1)$ is periodic with period $l(s+1)$.

Proof. Let $\left(x_{n}^{(1)}, x_{n}^{(2)}, \ldots, x_{n}^{(k)}\right)$ be a solution of system (2.1). Then $x_{n}^{i}$ is a solution of equation (2.5.i) for each $i$. The assumptions of the theorem imply that $g_{i}^{2}=g_{i}$ for each $i$. By lemma 2.3, we have

$$
\begin{aligned}
x_{(m+2)(s+1) l+r-s}^{(i)} & =g_{i}^{m+2}\left(x_{r-s}^{(i)}\right) \\
& =g_{i}^{m+1}\left(x_{r-s}^{(i)}\right) \\
& =x_{(m+1)(s+1) l+r-s}^{(i)} .
\end{aligned}
$$

Consequently, system (2.1) is periodic with period $l(s+1)$.

Theorem 2.8. Let $\alpha$ and $a$ be complex numbers. The system

$$
x_{n+1}^{(1)}=\frac{x_{n-s}^{(\pi(1))}+a}{\alpha x_{n-s}^{(\pi(1))}-1}, x_{n+1}^{(2)}=\frac{x_{n-s}^{(\pi(2))}+a}{\alpha x_{n-s}^{(\pi(2))}-1}, \ldots, x_{n+1}^{(k)}=\frac{x_{n-s}^{(\pi(k))}+a}{\alpha x_{n-s}^{(\pi(k))}-1},
$$

is periodic with period $l(s+1)$.

Proof. Set $f_{i}(x)=\frac{x+a}{\alpha x-1}, x \in \mathbb{C} \backslash\left\{\frac{1}{\alpha}\right\}, i \in \mathbb{Z}_{k}$. We can see that $f_{i}: \mathbb{C} \backslash\left\{\frac{1}{\alpha}\right\} \rightarrow \mathbb{C} \backslash\left\{\frac{1}{\alpha}\right\}$ is an involution for every $i$. By theorem 2.6, we insure that system $(2.9)$ is periodic with period $l(s+1)$. 
Theorem 2.9. The coupled system

$$
x_{n+1}=\frac{y_{n-s}+a}{\alpha_{1} y_{n-s}-1}, \quad y_{n+1}=\frac{x_{n-s}+a}{\alpha_{2} x_{n-s}-1}, n \in \mathbb{Z}^{\geq 0},
$$

where $\alpha_{2}=-\left(\frac{2}{a}+\alpha_{1}\right)$ and $a \neq 0$, is periodic with period $4(s+1)$.

Proof. One can see that $f_{i}(x)=\frac{x+a}{\alpha_{i} x-1}$ is a function from $\mathbb{C} \backslash\left\{\frac{1}{\alpha_{1}}, \frac{1}{\alpha_{2}},-\frac{a}{2}\right\}$ onto itself, $f_{1} f_{2}=f_{2} f_{1}=\frac{-x a}{2 x+a}$ and $f_{i}$ is involution for every $i$. Applying theorem 2.5, we arrive at the periodicity of the system with period $4(s+1)$.

\section{Illustrative examples}

(i) The system

$$
x_{n+1}=\frac{y_{n-s}+a}{\alpha y_{n-s}-1}, y_{n+1}=\frac{z_{n-s}+a}{\alpha z_{n-s}-1}, z_{n+1}=\frac{x_{n-s}+a}{\alpha x_{n-s}-1},
$$

is periodic with period $6(s+1)$. Indeed, the permutation which corresponds to this system is $\pi=\left(\begin{array}{lll}1 & 2 & 3\end{array}\right)$. So, its order is 3 . This implies that the system is periodic with period $6(s+1)$.

(ii) The system

$$
x_{n+1}=\frac{z_{n-s}+a}{\alpha z_{n-s}-1}, y_{n+1}=\frac{x_{n-s}+a}{\alpha x_{n-s}-1}, z_{n+1}=\frac{y_{n-s}+a}{\alpha y_{n-s}-1},
$$

is periodic with period $6(s+1)$. The permutation which corresponds to this system is $\pi=\left(\begin{array}{lll}1 & 3 & 2\end{array}\right)$. Again its order is 3 . This implies that the system is periodic with period $6(s+1)$.

(iii) The system

$$
x_{n+1}=\frac{z_{n-s}+a}{\alpha z_{n-s}-1}, y_{n+1}=\frac{w_{n-s}+a}{\alpha w_{n-s}-1}, z_{n+1}=\frac{y_{n-s}+a}{\alpha y_{n-s}-1}, w_{n+1}=\frac{x_{n-s}+a}{\alpha x_{n-s}-1}
$$

is periodic with period $4(s+1)$. Indeed, The permutation which describes this system is $\pi=\left(\begin{array}{llll}1 & 3 & 2 & 4\end{array}\right)$ with order 4. Then the periodicity of the system is $4(s+1)$.

(iv) The system

$$
x_{n+1}=\frac{y_{n-s}+a}{\alpha y_{n s}-1}, y_{n+1}=\frac{x_{n-s}+a}{\alpha x_{n-s}-1}, z_{n+1}=\frac{u_{n-s}+a}{\alpha u_{n-s}-1}, u_{n+1}=\frac{z_{n-s}+a}{\alpha z_{n-s}-1}
$$

is periodic with period $2(s+1)$. The permutation here is $\pi=\left(\begin{array}{lll}1 & 2\end{array}\right)\left(\begin{array}{ll}3 & 4\end{array}\right)$. So its order is 2 and thereby the system is periodic with period $2(s+1)$.

(v) The system

$$
x_{n+1}=\frac{z_{n}+a}{\alpha z_{n}-1}, y_{n+1}=\frac{x_{n}+a}{\alpha x_{n}-1}, z_{n+1}=\frac{u_{n}+a}{\alpha u_{n}-1}, u_{n+1}=\frac{y_{n}+a}{\alpha y_{n}-1}
$$

is periodic with period 4 .

(vi) The following matrices

$$
A_{1}=\left(\begin{array}{cc}
-a & \frac{1-a^{2}}{b} \\
b & a
\end{array}\right), b \neq 0
$$

and

$$
A_{2}=\left(\begin{array}{lll}
0 & 1 & 0 \\
1 & 0 & 0 \\
0 & 0 & 1
\end{array}\right)
$$


are involution matrices. Therefore the system

$$
x_{n+1}^{(1)}=A x_{n-s}^{(\pi(1))}, x_{n+1}^{(2)}=A x_{n-s}^{(\pi(2))}, \ldots, x_{n+1}^{(k)}=A x_{n-s}^{(\pi(k))},
$$

is periodic with period $l(s+1)($ resp. $2 l(s+1))$ when $l=0 \bmod 2(\operatorname{resp} . l=1 \bmod 2)$, where $\pi$ is a permutation of order $l$ and $A \in\left\{A_{1}, A_{2}\right\}$.

(vii) The following matrices

$$
A_{1}=\frac{1}{2}\left(\begin{array}{cc}
1-\cos \theta & \sin \theta \\
\sin \theta & 1+\cos \theta
\end{array}\right), \theta \in \mathbb{R}
$$

and

$$
A_{2}=\left(\begin{array}{ccc}
a & 1-a & 0 \\
a & 1-a & 0 \\
0 & 0 & 1
\end{array}\right)
$$

are idempotent matrices. Therefore the system

$$
x_{n+1}^{(1)}=A x_{n-s}^{(\pi(1))}, x_{n+1}^{(2)}=A x_{n-s}^{(\pi(2))}, \ldots, x_{n+1}^{(k)}=A x_{n-s}^{(\pi(k))},
$$

is periodic with period $l(s+1)($ resp. $2 l(s+1))$ when $l=0 \bmod 2($ resp. $l=1 \bmod 2)$, where $\pi$ is a permutation of order $l$ and $A \in\left\{A_{1}, A_{2}\right\}$.

\section{References}

[1] Oleg Bogopolski, Introduction to Group Theory, Cambridge University Press 1994.

[2] F. Albert Cotton, Chemical Applications of Group Theory, Third Edition, John Wiley \& Sons, New York,1990.

[3] S. Sternberg, Group Theory and Physics, Institute of Computer Science, Moscow-Izhevsk, 2008.

[4] I. Yalcinkaya, C. Cinar, and M. Atalay, On the Solutions of Systems of Difference Equations, Advances in Difference Equations Volume 2008, Article ID 143943, 9 pages doi:10.1155/2008/143943.

[5] T. W. Hungerford, Algebra, Springer, 1974.

[6] E. M. Elsayed, Alotaibi Abdullah, Almaylabi Hajar A, On the Periodicity and Solutions of Some Difference Equations Systems, Journal of Computational and Theoretical Nanoscience, Volume 13, Number 3, 1624-1628, 2016.

[7] C. S. Kubrusly, The elements of operator theory, second edition, 2011.

[8] G. Papaschinopoulos and C. J. Schinas, On a system of two nonlinear difference equations, Journal of Mathematical Analysis and Applications, vol. 219, no. 2, 415-426, 1998.

[9] Stevo Stevic, A. Mohammed Alghamdi, A. Maturi Dalal and Naseer Shahzad, On the Periodicity of Some Classes of Systems of Nonlinear Difference Equations, Abstract and Applied Analysis, Volume 2014 (2014), Article ID 982378, 6 pages. 


\title{
A NEW RESULT ON THE STABILITY OF A STOCHASTIC DIFFERENTIAL EQUATION OF THIRD-ORDER WITH A TIME-LAG
}

\author{
Ayman M. Mahmoud* \\ Department of Mathematics, Faculty of Science, New Valley Branch, \\ Assiut University, New Valley, El-Khargah 72111, Egypt \\ e-mail:math_ayman27@yahoo.com \\ Cemil Tunç** \\ Department of Mathematics, \\ Faculty of Science, Van Yuzuncu Yil University \\ 65080, Van-Turkey \\ e-mail:cemtunc@yahoo.com
}

Received 1/10/2017 $\quad$ Revised $\quad$ Accepted 21/11/2017

\begin{abstract}
Here, stochastic asymptotic stability (SAS) of the zero solution of a stochastic delay differential equation (SDDE) of third order such as

$$
\dddot{x}(t)+f(\dot{x}(t)) \ddot{x}(t)+g(\dot{x}(t-\tau))+h(x(t-\tau))+\sigma x(t) \dot{\omega}(t)=0
$$

is discussed. To arrive at the aim of this paper, a suitable Lyapunov functional (LF) is defined and then used to find conditions that guaranteeing the (SAS) of solutions. We give an example to verify the analysis made in this paper.
\end{abstract}

2010 Mathematics Subject Classification. 34K20, 34K50, 60H35

Keywords: (SAS), (LF), (SDDE), third-order.

\section{Introduction}

A stochastic differential equation (SDE) involves a variable which indicates random white noise calculated as the derivative of Brownian motion or the Wiener process. However, other types of random behaviors are possible, for example,jump processes. There are two dominating versions of stochastic calculus (SC), the Itô (SC) and the Stratonovich (SC). Both of these (SC)have advantages and disadvantages(see, for example, [1]) and conveniently, one can readily convert an Itô (SDE) to an equivalent Stratonovich (SDE) and back again.

The theory of (SDEs) has attracted considerable attention of many scholars in the last years. Therefore, it has extensively been studied in the relevant literature and there exists a large number of books, which provides full details of the background of probability theory and (SC), for example, see the books of [1], [2], [3], [4], [5], [6] and the references of these sources.

Since then the number of contributions to statistics, numerics and control theory of (SDEs) have been rapidly increasing. It is well known that stochastic models (SMs) have important roles in science and industry, where many authors can encounter with (SDEs).

\footnotetext{
*Correspondence: cemtunc@yahoo.com
} 
Therefore, (SMs) can have many important roles in various scientific areas such as biology, economy, medicine, engineering and so on (see,[7], [8], [9]). By this time, numerous kind of (LFs) have been used as basic tools to study qualitative problems in many deterministic/(SDEs) and (DDEs).

In addition, (SDDEs) represent a relatively new field of the qualitative theory of (DEs) and (DDEs). The significance of (SDDEs) has become more evident in recent years due to a great variety of their applications in modeling real world phenomena. Unfortunately, it is in general not possible to give or obtain analytic expressions for solutions of (SDEs) and (SDDEs). Therefore, most of the papers are interested in to characterize the qualitatively behaviors of solutions (QBSs) without solving (SDEs) and (SDDEs). To the best of our knowledge,up to now in the relative literature, the Lyapunov's theory is the most powerful tool for qualitative analysis of (SDEs)and (SDDEs) of higher order without solving that equations.

Hence, many papers dealt with the (QBSs)of higher order (DDEs) with (a or multiple) time lag(s) and obtained many interesting results by means of this theory, see, for example, the papers given in [10],[11], [12], [13], [14], [15], [16], [17], [18], [19], [20], [21] and the references cited therein to mention few). In deed, the many authors focused on (QBSs) of different models of (SDEs) and they got numerous interesting results over the last few years (see, for example, [22], [23], [24], [25], $[26],[27])$ and the references therein.

With respect to our observation, up to this moment, the corresponding problem for the stability (S) and boundedness (B) of solutions of higher order (SDDEs) have been studied in the literature by only a few researches, see, for instance, [28], [29], [30], [31], [32] and [33]. Therefore, it is worth discussing the (QBSs)of (SDDEs). In 2015, the authors of [30] discussed the (AS) of the zero solution of (SDDEs) such as

$$
\dddot{x}(t)+a_{1} \ddot{x}(t)+g_{1}\left(\dot{x}\left(t-r_{1}(t)\right)\right)+f_{1}(x(t))+\sigma_{1} x(t) \dot{\omega}(t)=0
$$

and

$$
\dddot{x}(t)+a_{2} \ddot{x}(t)+f_{2}(x(t)) \dot{x}(t)+f_{3}\left(\dot{x}\left(t-r_{2}(t)\right)\right)+\sigma x(t-h(t)) \dot{\omega}(t)=0 .
$$

In 2017, the author of [32] studied (S) and (B) of solutions of the (SDDEs) in the following form

$$
\dddot{x}(t)+a \ddot{x}(t)+b \dot{x}(t)+h(x(t-\tau))+\sigma x(t) \dot{\omega}(t)=p(t, x(t), \dot{x}(t), \ddot{x}(t)) .
$$

It should be noted that throughout the references of this papers, (LFs) have been used as a basic tool to investigate the (QBSs) mathematical modes therein.

Here, we take into consideration the (SDDE) of third order

$$
\dddot{x}(t)+f(\dot{x}(t)) \ddot{x}(t)+g(\dot{x}(t-\tau))+h(x(t-\tau))+\sigma x(t) \dot{\omega}(t)=0 .
$$

(SDDE) (1.1) can be written as

$$
\begin{aligned}
& \dot{x}=y, \\
& \dot{y}=z, \\
& \dot{z}=-f(y) z-g(y)-h(x)+\int_{t-\tau}^{t} g^{\prime}(y(s)) z(s) d s+\int_{t-\tau}^{t} h^{\prime}(x(s)) y(s) d s-\sigma x(t) \dot{\omega}(t),
\end{aligned}
$$

where $\sigma$ and $\tau$ are two positive constants; $f(\dot{x}), g(\dot{x})$ and $h(x)$ are continuous functions, $g(0)=f(0)=h(0)=0 ; \omega(t) \in \mathbb{R}^{m}$ is a $m$-dimensional standard Brownian motion or Wiener process defined on the probability space, a stochastic process representing the noise.

In this work, we aim to find new conditions for the (SAS) of the zero solution of(SDDE) (1.1) by defining an appropriate (LF).

Remark 1.1. We have the following observations:

(1) If $\sigma=0$ in (1.1), then we have a general (DDE) of third-order, which has been investigated extensively in relevant literature.

(2) Whenever $f(\dot{x}(t))=a$ and $g(\dot{x}(t-\tau))=b \dot{x}(t)$, where $a$ and $b$ are two positives constants, then (SDDE) (1.1) reduces to a (SDDE) of third-order discussed in [32]. Therefore Theorem 3.1 includes and extends the stability result discussed in [32]. 
(3) The obtained results in [28], [29], [31] and [33] are on (SDDEs) of second-order, but the obtained results in [30] and [32] are only on (SDDEs)of third order in the previous literature.

Motivated by references of this paper and the papers and books can found in the literature, we aim to do a contribution to the literature by obtaining a new result on the (SAS) of solutions of a new (SDDE) model, which has not been discussed in the literature yet.This fact is the novelty and originality of this article.

\section{Preliminary results}

Let $\omega(t)=\left(\omega_{1}(t), \ldots, \omega_{m}(t)\right)$ be an $m$-dimensional Brownian motion. Let us consider an $n$-dimensional (SDE)

$$
d x(t)=F(t, x(t)) d t+G(t, x(t)) d \omega(t) \quad \text { on } t \geq 0,
$$

with initial condition $x(0)=x_{0} \in \mathbb{R}^{n}$. We suppose that the functions $F: \mathbb{R}^{+} \times \mathbb{R}^{n} \rightarrow \mathbb{R}^{n}$ and $G: \mathbb{R}^{+} \times \mathbb{R}^{n} \rightarrow \mathbb{R}^{n \times m}$ are continuous and satisfy the Lipschitz condition (see, for example, [22],[26]). Hence, the existence and uniqueness of solutions of (SDE) (2.1) are guaranteedon $t \geq 0$, say $x\left(t ; x_{0}\right)$. In addition, we assume that $f(t, 0)=0$ and $g(t, 0)=0$.

We will show family of non-negative and differentiable functions $V\left(t, x_{t}\right)$ by $C^{1,2}\left(\mathbb{R}^{+} \times \mathbb{R}^{n} ; \mathbb{R}^{+}\right)$. That is, those functions once and twice continuously differentiable in $t$ and $x$, respectively.

Let us define an operator $\mathcal{L}$ on $C^{1,2}\left(\mathbb{R}^{+} \times \mathbb{R}^{n} ; \mathbb{R}^{+}\right)$by

$$
\mathcal{L} V\left(t, x_{t}\right)=V_{t}\left(t, x_{t}\right)+V_{x}\left(t, x_{t}\right) \cdot f(t, x)+\frac{1}{2} \operatorname{trace}\left[g^{T}(t, x) V_{x x}\left(t, x_{t}\right) g(t, x)\right],
$$

where

$$
V_{x}=\left(V_{x_{1}}, \ldots, V_{x_{n}}\right), V_{t}\left(t, x_{t}\right)=\frac{\partial V\left(t, x_{t}\right)}{\partial t}, V_{x}\left(t, x_{t}\right)=\left(\frac{\partial V\left(t, x_{t}\right)}{\partial x_{1}}, \ldots, \frac{\partial V\left(t, x_{t}\right)}{\partial x_{n}}\right)
$$

Furthermore,

$$
V_{x x}=\left(V_{x_{i} x_{j}}\right)_{n \times n}=\left(\frac{\partial^{2} V\left(t, x_{t}\right)}{\partial x_{i} \partial x_{j}}\right)_{n \times n}, \quad i, j=1, \ldots, n .
$$

Further, let $\mathcal{K}$ be the family of all nondecreasing and continuous functions $\vartheta: \mathbb{R}^{+} \rightarrow \mathbb{R}^{+}$with $\vartheta(0)=0$ and $\vartheta(r)>0$, if $r>0$.

Theorem 2.1. ([2], [4]) Assume that there exist $V \in C^{1,2}\left(\mathbb{R}^{+} \times \mathbb{R}^{n} ; \mathbb{R}^{+}\right)$and $\vartheta \in \mathcal{K}$ such that

(i) $V(t, 0)=0, \quad \vartheta(|x|) \leq V\left(t, x_{t}\right), \quad$ and

(ii) $\mathcal{L} V\left(t, x_{t}\right) \leq 0, \quad$ for all $(t, x) \in \mathbb{R}^{+} \times \mathbb{R}^{n}$.

Then the zero solution of the (SDE) is (SS).

Theorem 2.2. ([2], [4]) Assume that there exist $V \in C^{1,2}\left(\mathbb{R}^{+} \times \mathbb{R}^{n} ; \mathbb{R}^{+}\right)$and $\vartheta_{1}, \vartheta_{2}, \vartheta_{3} \in \mathcal{K}$ such that

(i) $\vartheta_{1}(|x|) \leq V\left(t, x_{t}\right) \leq \vartheta_{2}(|x|), \quad$ and

(ii) $\mathcal{L} V\left(t, x_{t}\right) \leq-\vartheta_{3}(|x|), \quad$ for all $(t, x) \in \mathbb{R}^{+} \times \mathbb{R}^{n}$.

Then the zero solution of the (SDE) is stochastically asymptotic stable in the large.

\section{Main result}

We now state the main (S) result for (SDDE) (1.1).

Theorem 3.1. Let

$\alpha, \beta, \gamma, a_{1}, a_{2}, L$ and $M$ be positive constants such that

(i) $h(0)=0, \frac{h(x)}{x} \geq \alpha>0$ and $h(x) \operatorname{sgn} x>0$, for all $x \neq 0$ and $\sup \left\{h^{\prime}(x)\right\}=\frac{\gamma}{2}$. 
(ii) $g(0)=0, \frac{g(y)}{y} \geq \beta>0$, for all $y \neq 0$.

(iii) $0 \leq f(y)-a_{1} \leq a_{2}$, for all $y$.

(iv) $\left|h^{\prime}(x)\right| \leq L$ and $\left|g^{\prime}(y)\right| \leq M$, for all $x, y$.

(v) $2 \alpha-2-a_{1}-\beta>\sigma^{2}$.

(vi) $a_{1} \beta-\gamma>2 \beta+6$.

Then the zero solution of (SDDE) (1.1) is stochastically asymptotic stable if

$$
\tau<\min \left\{\frac{2 \alpha-2-a_{1}-\beta-\sigma^{2}}{2(L+M)}, \frac{a_{1} \beta-\gamma-2 \beta-6}{4 \mu(L+M)+4 L(\mu+2)}, \frac{a_{1} \beta-\gamma-2 \beta}{4 \beta(L+M)+4 \beta M(\mu+2)}\right\},
$$

where

$$
\mu=\frac{a_{1} \beta+\gamma}{4 \beta} .
$$

Proof of Theorem 3.1. Define the (LF) $V(\cdot)=V\left(t, x_{t}, y_{t}, z_{t}\right)$ as

$$
\begin{aligned}
V(\cdot)= & \mu \int_{0}^{x} h(\xi) d \xi+h(x) y+\mu \int_{0}^{y} f(\eta) \eta d \eta+\int_{0}^{y} g(\eta) d \eta+\mu y z \\
& +\frac{1}{2} z^{2}+x z+x^{2}+\lambda \int_{-\tau}^{0} \int_{t+s}^{t} y^{2}(\theta) d \theta d s+\delta \int_{-\tau}^{0} \int_{t+s}^{t} z^{2}(\theta) d \theta d s,
\end{aligned}
$$

where $x_{t}=x(t+s), s \leq 0$ and $\lambda$ and $\delta$ are positive constants, and we determine them later.

Next, we have to show that $V\left(t, x_{t}, y_{t}, z_{t}\right)$ satisfies the assumptions of Theorem 2.2.

Moreover, by applying Itô formula in (3.1) using system (1.2), we find that

$$
\begin{aligned}
\mathcal{L} V(\cdot)= & h^{\prime}(x) y^{2}+2 x y+y z+\mu z^{2}-\mu g(y) y-f(y) z^{2}-f(y) x z-x g(y)-x h(x) \\
& +(\mu y+x+z)\left\{\int_{t-\tau}^{t} g^{\prime}(y(s)) z(s) d s+\int_{t-\tau}^{t} h^{\prime}(x(s)) y(s) d s\right\} \\
& +\lambda r y^{2}-\lambda \int_{t-\tau}^{t} y^{2}(s) d s+\delta r z^{2}-\delta \int_{t-\tau}^{t} z^{2}(s) d s+\frac{1}{2} \sigma^{2} x^{2} .
\end{aligned}
$$

Using assumptions $(i)-($ iii $)$ of Theorem 3.1, we get

$$
\begin{aligned}
\mathcal{L} V(\cdot) \leq & \frac{\gamma}{2} y^{2}+2 x y+y z+\mu z^{2}-\mu \beta y^{2}-a_{1} z^{2}-a_{1} x z-\beta x y-\alpha x^{2} \\
& +(\mu y+x+z)\left\{\int_{t-\tau}^{t} g^{\prime}(y(s)) z(s) d s+\int_{t-\tau}^{t} h^{\prime}(x(s)) y(s) d s\right\} \\
& +\lambda \tau y^{2}-\lambda \int_{t-\tau}^{t} y^{2}(s) d s+\delta \tau z^{2}-\delta \int_{t-\tau}^{t} z^{2}(s) d s+\frac{1}{2} \sigma^{2} x^{2} .
\end{aligned}
$$

Since $\left|h^{\prime}(x)\right| \leq L,\left|g^{\prime}(y)\right| \leq M$ and by using the inequality $2 u v \leq u^{2}+v^{2}$, we have

$$
\begin{gathered}
(\mu y+x+z) \int_{t-\tau}^{t} h^{\prime}(x(s)) y(s) d s \leq \frac{1}{2} \mu L \tau y^{2}+\frac{1}{2} L \tau x^{2}+\frac{1}{2} L \tau z^{2}+\left(\frac{1}{2} \mu+1\right) L \int_{t-\tau}^{t} y^{2}(s) d s, \\
(\mu y+x+z) \int_{t-\tau}^{t} g^{\prime}(y(s)) z(s) d s \leq \frac{1}{2} \mu M \tau y^{2}+\frac{1}{2} M \tau x^{2}+\frac{1}{2} M \tau z^{2}+\left(\frac{1}{2} \mu+1\right) M \int_{t-\tau}^{t} z^{2}(s) d s .
\end{gathered}
$$

Then, by substituting in (3.2), we find

$$
\mathcal{L} V(\cdot) \leq-\left(\frac{2 \alpha-2-a_{1}-\beta-\sigma^{2}}{2}-\frac{L+M}{2} \tau\right) x^{2}
$$




$$
\begin{aligned}
& -\left\{\mu \beta-\frac{\beta+\gamma+3}{2}-\frac{\mu(L+M)}{2} \tau-\lambda \tau\right\} y^{2} \\
& -\left(-\mu+\frac{a_{1}}{2}-\frac{1}{2}-\frac{L+M}{2} \tau-\delta r\right) z^{2} \\
& -\left(\lambda-\frac{\mu L}{2}-L\right) \int_{t-\tau}^{t} y^{2}(s) d s-\left(\delta-\frac{\mu M}{2}-M\right) \int_{t-\tau}^{t} z^{2}(s) d s .
\end{aligned}
$$

If we take

$$
\lambda=\frac{L(\mu+2)}{2}>0 \quad \text { and } \quad \delta=\frac{M(\mu+2)}{2}>0,
$$

then it is easy to see that

$$
\begin{aligned}
\mathcal{L} V(\cdot) \leq & -\left(\frac{2 \alpha-2-a_{1}-\beta-\sigma^{2}}{2}-\frac{L+M}{2} \tau\right) x^{2} \\
& -\left\{\frac{a_{1} \beta-\gamma-2 \beta-6}{4}-\frac{\mu(L+M)+L(\mu+2)}{2} \tau\right\} y^{2} \\
& -\left(\frac{a_{1} \beta-\gamma-2 \beta}{4 \beta}-\frac{L+M+M(\mu+2)}{2} \tau\right) z^{2} .
\end{aligned}
$$

Thus, if

$$
\tau<\min \left\{\frac{2 \alpha-2-a_{1}-\beta-\sigma^{2}}{2(L+M)}, \frac{a_{1} \beta-\gamma-2 \beta-6}{4 \mu(L+M)+4 L(\mu+2)}, \frac{a_{1} \beta-\gamma-2 \beta}{4 \beta(L+M)+4 \beta M(\mu+2)}\right\} .
$$

Hence, we can obtain such that

$$
\mathcal{L} V(\cdot) \leq-\rho_{1}\left(x^{2}+y^{2}+z^{2}\right), \quad \text { for some constant } \rho_{1}>0 .
$$

It is clear from assumptions $f(y) \geq a_{1}$ and $g(y) \geq \beta y$, and from conditions (ii) and (iii) of Theorem 3.1 that

$$
V(\cdot) \geq \mu \int_{0}^{x} h(\xi) d \xi+h(x) y+\frac{1}{2} \mu a_{1} y^{2}+\frac{1}{2} \beta y^{2}+\mu y z+\frac{1}{2} z^{2}+x z+x^{2} .
$$

It follows that

$$
\begin{aligned}
V(\cdot) \geq & \mu \int_{0}^{x} h(\xi) d \xi+\frac{1}{2 \beta}(\beta y+h(x))^{2}-\frac{1}{2 \beta} h^{2}(x)+\frac{1}{2} \mu a_{1} y^{2}+\mu y z \\
& +\left(x+\frac{z}{2}\right)^{2}+\frac{1}{4} z^{2} \\
= & \frac{1}{2 \beta}(\beta y+h(x))^{2}+\left(\mu y+\frac{z}{2}\right)^{2}+\frac{1}{2} \mu\left(a_{1}-2 \mu\right) y^{2}+\left(x+\frac{z}{2}\right)^{2} \\
& +\frac{1}{2 \beta y^{2}}\left[4 \int_{0}^{x} h(\xi)\left\{\int_{0}^{y}\left(\mu \beta-h^{\prime}(x)\right) \eta d \eta\right\} d \xi\right] ; \quad y \neq 0 .
\end{aligned}
$$

Now we recall that

$$
\begin{aligned}
& a_{1}-2 \mu=a_{1}-2\left(\frac{a_{1} \beta+\gamma}{4 \beta}\right)=\frac{a_{1} \beta-\gamma}{2 \beta}>0, \\
& \mu \beta-h^{\prime}(x) \geq \frac{a_{1} \beta+\gamma}{4 \beta}-\frac{\gamma}{2}=\frac{a_{1} \beta-\gamma}{4}>0 .
\end{aligned}
$$

Therefore we find

$$
\begin{aligned}
& \frac{1}{2 \beta y^{2}}\left[4 \int_{0}^{x} h(\xi)\left\{\int_{0}^{y}\left(\mu \beta-h^{\prime}(x)\right) \eta d \eta\right\} d \xi\right] \\
& \quad \geq \frac{1}{2 \beta y^{2}}\left[4 \int_{0}^{x} h(\xi)\left\{\int_{0}^{y}\left(\frac{a_{1} \beta-\gamma}{4}\right) \eta d \eta\right\} d \xi\right]=\frac{a_{1} \beta-\gamma}{4 \beta} \int_{0}^{x} h(\xi) d \xi
\end{aligned}
$$


which together with (3.4), implies the following inequality

$$
\begin{aligned}
V(\cdot) \geq & \frac{1}{2 \beta}(\beta y+h(x))^{2}+\left(\mu y+\frac{z}{2}\right)^{2}+\frac{1}{2} \mu\left(\frac{a_{1} \beta-\gamma}{2 \beta}\right) y^{2}+\left(x+\frac{z}{2}\right)^{2} \\
& +\frac{a_{1} \beta-\gamma}{4 \beta} \int_{0}^{x} h(\xi) d \xi .
\end{aligned}
$$

Then

$$
V(\cdot) \geq \rho_{2}\left(x^{2}+y^{2}+z^{2}\right), \quad \text { for some constant } \rho_{2}>0 .
$$

Now since $f(y) \leq a_{1}+a_{2},\left|h^{\prime}(x)\right| \leq L$ and $\left|g^{\prime}(y)\right| \leq M$, then, by using the mean-value theorem for the derivatives, we can find $h(x) \leq L x$ and $g(y) \leq M y$. Therefore we can re-arrange (3.1) such as the following form

$$
\begin{aligned}
V(\cdot) \leq & \frac{1}{2} \mu L x^{2}+L x y+\frac{1}{2} \mu\left(a_{1}+a_{2}\right) y^{2}+\frac{1}{2} M y^{2}+\mu y z+\frac{1}{2} z^{2} \\
& +x z+x^{2}+\lambda \int_{-\tau}^{0} \int_{t+s}^{t} y^{2}(\theta) d \theta d s+\delta \int_{-r}^{0} \int_{t+s}^{t} z^{2}(\theta) d \theta d s .
\end{aligned}
$$

On the other hand, it is obvious that

$$
\begin{aligned}
& \int_{-\tau}^{0} \int_{t+s}^{t} y^{2}(\theta) d \theta d s \leq\|y\|^{2} \int_{t-\tau}^{t}(\theta-t+\tau) d \theta=\frac{\tau^{2}}{2}\|y\|^{2}, \\
& \int_{-\tau}^{0} \int_{t+s}^{t} z^{2}(\theta) d \theta d s \leq\|z\|^{2} \int_{t-\tau}^{t}(\theta-t+\tau) d \theta=\frac{\tau^{2}}{2}\|z\|^{2} .
\end{aligned}
$$

So that by using the pervious inequalities in (3.6) and the estimate $u v \leq \frac{1}{2}\left(u^{2}+v^{2}\right)$, we have

$$
\begin{aligned}
V(\cdot) & \leq \frac{1}{2}\{(\mu+1) L+3\}\|x\|^{2}+\frac{1}{2}\left\{\left(a_{1}+a_{2}+1\right) \mu+M+L+\lambda \tau^{2}\right\}\|y\|^{2} \\
& +\frac{1}{2}\left(\mu+2+\delta \tau^{2}\right)\|z\|^{2} .
\end{aligned}
$$

Then there exists a positive constant $\rho_{3}$ satisfying the following

$$
V(\cdot) \leq \rho_{3}\left(x^{2}+y^{2}+z^{2}\right), \quad \text { for some } \rho_{3}>0 .
$$

Therefore, from the results (3.3), (3.5) and (3.7), we see that all the conditions of Theorem 2.2 hold, and hence the zero solution of (1.1) is stochastically asymptotic stable.

This completes the proof of the last theorem.

\section{Example}

In this section we provide an example to validate the theorem proved in the former section.

Example 1: Consider the below (SDDE) of third order as follows:

$$
\dddot{x}+\left(8+\frac{1}{1+\dot{x}^{2}}\right) \ddot{x}+8 \dot{x}(t-\tau)+\sin (\dot{x}(t-\tau))+12 x(t-\tau)+\frac{x(t-\tau)}{1+x^{2}(t-\tau)}+2 x(t) \dot{\omega}(t)=0 .
$$

Its equivalent system is given as

$$
\begin{aligned}
\dot{x}= & y \\
\dot{y}= & z \\
\dot{z}= & -\left(8+\frac{1}{1+y^{2}}\right) z-(8 y+\sin y)+\int_{t-\tau}^{t}\{8+\cos y(s)\} z(s) d s \\
& \quad-\left(12 x+\frac{x}{1+x^{2}}\right)+\int_{t-\tau}^{t}\left\{12+\frac{1-x^{2}(s)}{\left(1+x^{2}(s)\right)^{2}}\right\} y(s) d s-2 x(t) \dot{\omega}(t) .
\end{aligned}
$$


It follows that (SDDE) (4.1) is a special case of (SDDE) (1.1), and when we compare (SDDE) (4.1) with (SDDE)(1.1) we obtain the following relations:

$$
f(y)=8+\frac{1}{1+y^{2}}, \quad \text { since } \quad 0 \leq \frac{1}{1+y^{2}} \leq 1,
$$

it tends to $a_{1}=8$, and $a_{2}=1$. Also since

$$
g(y)=8 y+\sin y, \quad \frac{g(y)}{y}-8=\frac{\sin y}{y},
$$

therefore

$$
\begin{gathered}
-1 \leq \frac{g(y)}{y}-8 \leq 1 \\
g^{\prime}(y)=8+\cos y, \quad \text { for } \quad|8+\cos y| \leq 9 .
\end{gathered}
$$

Then we can take $\beta=7$ and $M=9$. Since

$$
h(x)=12 x+\frac{x}{1+x^{2}}, \text { it follows that } \frac{h(x)}{x} \geq 12 .
$$

Then, we get

$$
\left|h^{\prime}(t)-12\right| \leq 1 \text {, then }\left|h^{\prime}(t)\right| \leq 13,
$$

hence we can take $\alpha=12$ and $L=14$.

Next, we can note that $\sup \left\{\left|h^{\prime}(x)\right|\right\}=13$, then we can take $\gamma=26$.

By noting the former discussion, we see that

$$
\mu=\frac{a_{1} \beta+\gamma}{4 \beta} \cong 2.93, \quad a_{1} \beta-\gamma-2 \beta-6=10 ; \quad 2 \alpha-2-a_{1}-\beta=7>\sigma^{2}=4 .
$$

Thus, we can note that all assumptions $(i)-(i v)$ of Theorem 3.1 hold true.

This result implies that the zero solution of (SDDE) (4.1) is stochastically asymptotic stable if $\tau=0.008$.

\section{References}

[1] B. Øksendal, Stochastic Differential Equations (An Introduction with Applications), Springer-Verlag, Heidelberg-New York, 2000.

[2] L. Arnold, Stochastic Differential Equation: Theory and Applications, John and Sons, 1974.

[3] K. Liu, Stability of Infinite Dimensional Stochastic Differential Equation with Applications, Boca Raton, Chapman Hall/CRC, 2006.

[4] X. Mao, Stochastic Differential Equations and Their Applications, Horwood Publishing, Chichester, UK, 1997.

[5] S.-E.A. Mohammed, Stochastic Functional Differential Equations, Research Notes in Mathematics 99, Pitman Advanced Publishing Program, Boston-London-Melbourne, 1984.

[6] U.H. Thygesen, A Survey of Lyapunov Techniques for Stochastic Differential Equations, IMM Technical Report nc., 1997.

[7] P. Tass, Phase Resetting in Medicine and Biology: Stochastic Modelling and Data Analysis, Berlin, Springer-Verlag, 1999.

[8] J. Lei, M.C. Mackey, Stochastic differential delay equation: moment stability and application to hematopoietic stem cell regulation system, SIAM J. Appl. Math., 67(2007) 387-407.

[9] T. Tian, K. Burrage, P.M. Burrage and M. Carletti, Stochastic delay differential equations for genetic regulatory networks, J. Comput. Appl. Math., 205(2007) 696-707. 
[10] A.T. Ademola and P.O. Arawomo, Uniform stability and boundedness of solutions of nonlinear delay differential equations of the third-order, Mathematical Journal Okayama University, 55(2013), 157-166.

[11] A.T. Ademola, B.S. Ogundare, M.O. Ogundiran and O.A. Adesina, Stability, boundedness and existence of periodic solutions to certain third-order delay differential equations with multiple deviating arguments, International Journal of Differential Equations, 2015(2015), 1-12.

[12] A.M. Mahmoud, On the asymptotic stability of solutions for a certain non-autonomous third-order delay differential equation, British Journal of Mathematics and Computer Science, 16(3)(2016) 1-12.

[13] M.O. Omeike, New results on the stability of solution of some non-autonomous delay differential equations of the third-order, Differential Equations and Control Processes, 1(2010) 18-29.

[14] L. Oudjedi, D. Beldjerd and M. Remili, On the stability of solutions for non-autonomous delay differential equations of third-order, Differential Equations and Control Processes, 1(2014) 22-34.

[15] A.I. Sadek, On the stability of solutions of some non-autonomous delay differential equations of the third-order, Asymptotic Anal., 43(2005) 1-7.

[16] C. Tunç, On the stability of solutions for non-autonomous delay differential equations of third-order, Iran. J. Sci. Technol. A, 32(2008) 261-273.

[17] C. Tunç, On the stability and boundedness of solutions of nonlinear third-order differential equations with delay, Filomat, 24(3)(2010) 1-10.

[18] C. Tunç, A note on the stability and boundedness of non-autonomous differential equations of second-order with a variable deviating argument, Afr. Math., 25(2)(2014) 417-425.

[19] C. Tunç, Global stability and boundedness of solutions to differential equations of third-order with multiple delays, Dynam. Systems Appl., 24(2015) 467-478.

[20] C. Tunç, On the stability and boundedness of certain third-order non-autonomous differential equations of retarded type, Proyecciones, 34(2)(2015) 147-159.

[21] Y. Zhu, On stability, boundedness and existence of periodic solution of a kind of third-order nonlinear delay differential system, Ann. Differential Equations, 8(2)(1992) 249-259.

[22] X. Mao, Existence and uniqueness of solutions of stochastic integral equations, J. Fuzhou Univ., 4(1983) 41-50.

[23] X. Mao, Attraction, stability and boundedness for stochastic differential delay equations, Nonlinear Analysis, 47(2001) 4795-4806.

[24] X. Mao and A. Shah, Exponential stability of stochastic differential delay equations, Stochastics and Stochastic Reports, 60(1997) 135-153.

[25] X. Mao, C. Yuan and J. Zou, Stochastic differential delay equations of population dynamics, J. Math. Anal. Appl., 304(2005) 296-320.

[26] R. Wu and X. Mao, Existence and uniqueness of solutions of stochastic differential equations, Stochastics, 11(1983) 19-32.

[27] F. Wu and S. Hu, Attraction, stability and robustness for stochastic functional differential equations with infinite delay, Automatica, 47(2011) 2224-2232.

[28] A.M.A. Abou-El-Ela, A.I. Sadek and A.M. Mahmoud, On the stability of solutions for certain second-order stochastic delay differential equations, Differential Equations and Control Processes, 2(2015) 1-13.

[29] A.M.A. Abou-El-Ela, A.I. Sadek, A.M. Mahmoud and R.O.A. Taie, On the stochastic stability and boundedness of solutions for stochastic delay differential equation of the second-order, Chin. J. Math., 2015(2015) 1-8. 
[30] A.M.A. Abou-El-Ela, A.I. Sadek, A.M. Mahmoud and E.S. Farghaly, Stability of solutions for certain third-order nonlinear stochastic delay differential equations, Ann. Differential Equations, 31(2015) 253-261.

[31] A.M.A. Abou-El-Ela, A.I. Sadek, A.M. Mahmoud and E.S. Farghaly, Asymptotic stability of solutions for a certain non-autonomous second-order stochastic delay differential equation, Turk. J. Math., 41(2)(2017) 576-584.

[32] A.T. Ademola, Stability, boundedness and uniqueness of solutions to certain third-order stochastic delay differential equations, Differential Equations and Control Processes, 2(2017) 24-50.

[33] A.T. Ademola, S. Moyo, B.S. Ogundare, M.O. Ogundiran and O.A. Adesina, Stability and boundedness of solutions to a certain second-order non-autonomous stochastic differential equation, International Journal of Analysis, 2016(2016), 1-11. 